\title{
Gauge Field Theories on a $\perp$ lattice
}

\author{
Matthias Burkardt \\ New Mexico State University \\ Las Cruces, NM 88003, U.S.A.
}

\begin{abstract}
In these notes, the transverse $(\perp)$ lattice approach is presented as a means to control the $k^{+} \rightarrow 0$ divergences in light-front QCD. Technical difficulties of both the canonical compact formulation as well as the non-compact formulation of the $\perp$ lattice motivate the color-dielectric formulation, where the link fields are linearized.
\end{abstract}

\section{INTRODUCTION}

The main subject of these notes are difficulties associated with the formulation of gauge field theories on a transverse $(\perp)$ lattice using light-front $(\mathrm{LF})$ quantization. Because of these difficulties, the reader may wonder about the advantages of this approach - particularly given the successes of Euclidean lattice gauge theory (LGT). The primary motivations for formulating QCD in this framework is that LF quantization is the most physical approach towards a microscopic description of the parton distributions measured in deep-inelastic scattering as well as many other hard processes. ${ }^{1}$ It is important to emphasize this fact in this brief introduction since it explains why LF quantization of QCD and the $\perp$ lattice should be investigated as a possible alternative to Euclidean and Hamiltonian LGT formulations - despite the difficulties that will be discussed in the remainder of these notes.

\section{Why LF gauge?}

Although the choice of quantization hyperplane and the choice of gauge are in principle independent issues, the so-called LF gauge $\left(A^{+}=0\right)$ turns out to be highly preferable for the canonical formulation of LFQCD. The main reason is that in the kinetic energy term for $\vec{A}_{\perp}\left(\right.$ from $\left.-\frac{1}{4} F^{\mu \nu} F_{\mu \nu}\right)$

$$
\mathcal{L}_{k i n, A_{\perp}}=D_{+} \vec{A}_{\perp} D_{-} \vec{A}_{\perp}=\left(\partial_{+}-i g A_{+}\right) \vec{A}_{\perp}\left(\partial_{-}-i g A_{-}\right) \vec{A}_{\perp},
$$

the term multiplying the 'time' derivative of $\vec{A}_{\perp}$ (i.e. $\partial_{+} \vec{A}_{\perp}$ ) contains also $A_{-}=A^{+}$. Therefore, the canonical momenta

1) This and other motivations are discussed in more detail in Ref. [1] and in references therein. 


$$
\Pi=\frac{\partial \mathcal{L}}{\partial\left(\partial_{+} A_{\perp}\right)}=\left(\partial_{-}-i g A_{-}\right) A_{\perp},
$$

which are the LF analog to $\Pi=\frac{\partial \mathcal{L}}{\partial\left(\partial_{0} A_{\perp}\right)}$ in equal time quantization, are "simple" (i.e. linear in the fields) if and only if $A_{-}=A^{+}=0$. Therefore, in order to avoid having to deal with a system that has to satisfy nonlinear constraints ${ }^{2}$ one normally selects $A^{+}=0$ gauge before quantizing in LF coordinates.

However, this choice of gauge is not entirely free of problems. To illustrate this fact, let us start from the Euler-Lagrange equation for $A^{-}$in QED ${ }^{3}$

$$
-\partial_{-}^{2} A^{-}=g J^{+}+\partial_{-} \vec{\partial}_{\perp} \vec{A}_{\perp} \equiv g \tilde{J}^{+}
$$

(the LF analog to the Poisson equation), which is also a constraint equation. It is convenient to eliminate $A^{-}$, using the solution to Eq.(3), i.e. $A^{-}\left(x^{-}, \vec{x}_{\perp}\right)=$ $-\frac{g}{2} \int_{-\infty}^{\infty} d y^{-}\left|x^{-}-y^{-}\right| \tilde{J}^{+}\left(y^{-}, \vec{x}_{\perp}\right)$, yielding an instantaneous interaction term

$$
V^{i n s t}=-\frac{g^{2}}{4} \int_{-\infty}^{\infty} d x^{-} \int_{-\infty}^{\infty} d y^{-} \int d^{2} x_{\perp} \tilde{J}^{+}\left(x^{-}, \vec{x}_{\perp}\right)\left|x^{-}-y^{-}\right| \tilde{J}^{+}\left(y^{-}, \vec{x}_{\perp}\right) .
$$

This linearly rising interaction in the LF Hamiltonian causes IR divergences, unless

$$
\int_{-\infty}^{\infty} d x^{-} \tilde{J}^{+}\left(x^{-}, \vec{x}_{\perp}\right)=0 \quad \forall \vec{x}_{\perp} .
$$

The origin of this problem lies in the fact that setting $A^{+}=0$ does not completely fix the gauge freedom. An $x^{-}$independent gauge transformation $A^{\mu} \longrightarrow \mathcal{U}^{\dagger} A^{\mu} \mathcal{U}-$ $\frac{i}{g} \mathcal{U}^{\dagger} \partial^{\mu} \mathcal{U}$, with $\mathcal{U}=\mathcal{U}\left(\vec{x}_{\perp}\right)$, leaves $A^{+}=0$ unchanged and Eq. (5) is just the Gauß' law constraint associated with this residual gauge symmetry ${ }^{4}$. As long as $\vec{x}_{\perp}$ is a continuous variable, Eq. (5) implies an infinite number of constraint on the states ( $\infty$ number of $\vec{x}_{\perp}$ !), which is again difficult to deal with. This is one of the motivations for discretizing the $\perp$ space direction in the context of LF quantization.

\section{THE TRANSVERSE LATTICE}

The basic idea behind the $\perp$ lattice [2] is to work in two continuous $\left(x^{0}\right.$ and $x^{3}$ or $x^{+}$and $\left.x^{-}\right)$space time directions and two discrete $\left[\vec{x}_{\perp} \equiv\left(x_{1}, x_{2}\right)\right]$ space directions, i.e. space-time consists of a 2-dimensional array of 2-dimensional sheets. ${ }^{5}$ The motivation for working with such a 'hybrid' formulation is that the discretized $\perp$ directions provide a the possibility to introduce a gauge invariant cutoff, while the

2) Eq. (2) is a constraint equation since it involves no time-derivative.

3) Since the problem that we are going to discuss occurs already in QED, we will discuss it there because of the simpler algebra.

4) Fixing the remaining gauge freedom requires dealing with explicit zero-mode degrees of freedom and it is still not completely understood how to do this!

5) In the closely related Hamiltonian LGT space-time consists of a 3-dim. array of 1-dim. lines. 
continuous longitudinal directions allow to maintain manifest longitudinal boost invariance (which is one of the advantages of the LF formulation).

The natural way to introduce gauge fields within this framework seems to be to work with compact link-fields $U_{\perp} \in S U\left(N_{C}\right)$ in the discretized $\perp$ directions (as is done in conventional LGT) and with non-compact gauge fields $A^{ \pm}$in the continuous longitudinal directions. It should be emphasized that both the $U_{\perp}$ 's as well as the $A^{ \pm}$(which are defined on the links and sites of the $\perp$ lattice respectively), are functions of two discrete and two continuous variables, i.e. one can think of the $\perp$ lattice action as consisting of many $1+1 \mathrm{dim}$. gauge theories coupled together.

The trouble with this formulation is the nonlinear $U_{\perp} \in S U\left(N_{C}\right)$ constraint on the link fields. The reason that this constraint is more difficult to handle on the $\perp$ lattice than in Euclidean or Hamiltonian LGT is due to the fact that the $U_{\perp} \mathrm{S}$ are still two dimensional fields (and not just variables, as in Euclidean LGT, or quantum mechanical rotors, as in Hamiltonian LGT). Despite several attempts in this direction [3], nobody has been able to construct a Fock space basis out of these "nonlinear $\sigma$ model" degrees of freedom which still allows one to evaluate matrix elements of the LF Hamiltonian.

Two possibilities to avoid the problems associated with the $S U(N)$-constraint have been pursued: The first is to work with non-compact gauge fields also in the $\perp$ direction and the other is to keep compact fields, but to relax the $S U(N)$ constraint and linearize the degrees of freedom.

\section{Non-compact formulation of the $\perp$ lattice}

Again, we illustrate the main difficulties in the context of QED. In order to satisfy the $U(1)$ constraint, one starts with the ansatz $U_{\perp}=\exp \left(i e A_{\perp}\right)$, yielding

$$
P^{-} \sim-\frac{1}{4} \sum_{\vec{n}_{\perp}} \int d x^{-} \int d y^{-} \tilde{j}^{+}\left(x^{-}, \vec{n}_{\perp}\right)\left|x^{-}-y^{-}\right| \tilde{j}^{+}\left(y^{-}, \vec{n}_{\perp}\right)+P_{\text {plaq }}^{-},
$$

where $P_{\text {plaq }}^{-}$is the $\perp$ plaquette interaction ( $x y$ orientation) and

$$
\tilde{j}^{+}\left(x^{-}, \vec{n}_{\perp}\right)=j_{q}^{+}\left(x^{-}, \vec{n}_{\perp}\right)+\frac{1}{e} \Delta_{\perp} \partial_{-} A_{\perp},
$$

where $\Delta_{\perp}$ is the discrete approximation to the $\perp$ Laplace operator and $j_{q}^{+}$is the portion of the current carried by the fermions.

As long as one restricts oneself to gauge fields with local fluctuations only (as in the Fock expansion!) one finds $A_{\perp}(+\infty)=A_{\perp}(-\infty)$ and thus

$$
\int_{-\infty}^{\infty} d x^{-\tilde{j}^{+}}\left(x^{-}, \vec{n}_{\perp}\right)=\int_{-\infty}^{\infty} d x^{-} j_{q}^{+}\left(x^{-}, \vec{n}_{\perp}\right)
$$

Together with Gauß' law this implies that $\int_{-\infty}^{\infty} d x^{-} j_{q}^{+}\left(x^{-}, \vec{n}_{\perp}\right)=0$, i.e. charges must add up to zero at each site! Transversely separated charges are only allowed in the 
presence of "soliton-like" gauge fields with $A_{\perp}\left(x^{-}=+\infty\right)-A_{\perp}\left(x^{-}=-\infty\right)=k e$, where $k$ is an integer! The physics behind this result becomes clear by noting that $\exp \left(i e A_{\perp}\right)$ acts like such a soliton operator: from the canonical commutation relations $\left[A_{\perp}\left(x^{-}\right), A_{\perp}\left(y^{-}\right)\right]=\frac{i}{2} \varepsilon\left(x^{-}-y^{-}\right)$, together with $\left[e^{A}, B\right]=[A, B] e^{A}$ if $[A, B]$ is a c-number, one finds

$$
\left[A_{\perp}\left(x^{-}=+\infty\right)-A_{\perp}\left(x^{-}=-\infty\right), \exp \left(i e A_{\perp}\left(y^{-}\right)\right)\right]=-e \exp \left(i e A_{\perp}\left(y^{-}\right)\right) .
$$

Therefore the Gauß' law constraint is satisfied if transversely separated charges are separated by a string of exponentials — just as one would have expected from gauge invariance - and the good news is that the infrared divergences cancel if states are constructed in a gauge invariant way.

It is instructive to examine in detail how the $k^{+} \rightarrow 0$ divergences cancel in $Q E D_{2+1}$. In $2+1$ dimensions, there is only one $\perp$ direction and therefore purely $\perp$ plaquette terms are absent. As a result, the whole dynamics of $A_{\perp}$ is described by its coupling to $A^{-}$and pure gauge, coupled to external sources, becomes exactly solvable in the non-compact formulation.

The rest frame energy of an external source $j^{+}$in $Q E D_{2+1}$ on a $\perp$ lattice is given by $H_{R F}=v^{+} P^{-}+H_{\text {recoil }}$, where $v^{+}$is the velocity of the source and

$$
P^{-}=\frac{e^{2} a}{2} \sum_{n} \int_{-\infty}^{\infty} d q^{+} \tilde{j}_{n}\left(q^{+}\right) \frac{1}{{q^{+2}}^{2}} \tilde{j}_{n}\left(-q^{+}\right) .
$$

is the instantaneous interaction arising from eliminating $A^{-}$and

$$
H_{\text {recoil }}=\frac{1}{2 v^{+}} \sum_{n} \int_{0}^{\infty} d k^{+} a_{n}^{\dagger}\left(k^{+}\right) a_{n}\left(k^{+}\right) k^{+}
$$

is a recoil term which appears in the LF description of fixed sources [4]. The effective current $\tilde{j}^{+}$receives contributions from both $A_{\perp}$ and the external current $j^{+}$[see also Eq.(7)]. In momentum space, one finds for the current on the $n^{\text {th }}$ site

$$
\tilde{j}_{n}^{+}\left(q^{+}\right)=e j_{n}^{+}\left(q^{+}\right)+\frac{i q^{+}}{a}\left[A_{\perp, n}\left(q^{+}\right)-A_{\perp, n-1}\left(q^{+}\right)\right],
$$

where we define the $n^{t h}$ site to be the one between the $(n-1)^{t h}$ and the $n^{\text {th }}$ link. It is instructive to decompose the instantaneous interaction into terms quadratic in $A_{\perp}$ and $j^{+}$respectively and a mixed term, i.e. $P^{-}=P_{A A}^{-}+P_{j j}^{-}+P_{J A}^{-}$, where

$$
\begin{aligned}
P_{A A}^{-} & =\sum_{n} \int_{0}^{\infty} \frac{d k^{+}}{2 a^{2} k^{+}}\left[a_{n}^{\dagger}\left(k^{+}\right)-a_{n-1}^{\dagger}\left(k^{+}\right)\right]\left[a_{n}\left(k^{+}\right)-a_{n-1}\left(k^{+}\right)\right] \\
& =\frac{4}{a^{2}} \int_{0}^{\infty} \frac{d k^{+}}{2 k^{+}} \int_{-\pi / a}^{\pi / a} d k_{\perp} \sin ^{2}\left(\frac{a k_{\perp}}{2}\right) a^{\dagger}\left(k^{+}, k_{\perp}\right) a\left(k^{+}, k_{\perp}\right) .
\end{aligned}
$$

where $a_{n}\left(q^{+}\right)=\int_{-\pi / a}^{\pi / a} \frac{d q_{\perp}}{\sqrt{2 \pi}} a\left(q^{+}, q_{\perp}\right) \exp \left(i q_{\perp}\right.$ an $)$. Furthermore 


$$
P_{j j}^{-}=\frac{e^{2} a}{2} \sum_{n} \int_{-\infty}^{\infty} d q^{+} j_{n}\left(q^{+}\right) \frac{1}{q^{+2}} j_{n}\left(-q^{+}\right)
$$

is the self-energy of the source, and the coupling of the source to $A_{\perp}$ reads

$$
\begin{aligned}
P_{j A}^{-} & =-i e \sum_{n} \int_{-\infty}^{\infty} \frac{d q^{+}}{q^{+}} j_{n}\left(q^{+}\right)\left[\tilde{A}_{n}\left(q^{+}\right)-\tilde{A}_{n-1}\left(q^{+}\right)\right] \\
& =\frac{i e}{\sqrt{2 a}} \int_{0}^{\infty} \frac{d q^{+}}{q^{+3 / 2}}\left\{j_{n}^{+}\left(q^{+}\right)\left[a_{n}\left(q^{+}\right)-a_{n-1}\left(q^{+}\right)\right]-j_{n}^{+}\left(-q^{+}\right)\left[a_{n}^{\dagger}\left(q^{+}\right)-a_{n-1}^{\dagger}\left(q^{+}\right)\right]\right\} .
\end{aligned}
$$

In order to calculate the self-energy of an external charge-distribution $j_{q}\left(q^{+}\right)$to order $e^{2}$, one needs to add the instantaneous self-interaction (10) [which is of $\mathcal{O}\left(e^{2}\right)$ already] in first order to the contribution from the coupling to $A_{\perp}$ (15) [which is only $\mathcal{O}(e)]$ treated in $2^{\text {nd }}$ order perturbation theory. The latter yields

$$
\delta E^{(2)}=-\frac{v^{+2} e^{2}}{a^{2}} \int_{0}^{\infty} \frac{d q^{+}}{q^{+^{3}}} \int_{-\pi / a}^{\pi / a} d q_{\perp} \frac{2 \sin ^{2}\left(\frac{a q_{\perp}}{2}\right) \tilde{j}(q) \tilde{j}(-q)}{\frac{q^{+}}{2 v^{+}}+\frac{2 v^{+}}{a^{2} q^{+}} \sin ^{2}\left(\frac{a q_{\perp}}{2}\right)},
$$

where we used the shorthand notation $j(q) \equiv j\left(q^{+}, q_{\perp}\right)$ and where $j_{n}\left(q^{+}\right)=$ $\int_{-\pi / a}^{\pi / a} \frac{d q_{\perp}}{\sqrt{2 \pi}} j\left(q^{+}, q_{\perp}\right) \exp \left(i q_{\perp} a n\right)$. In general, $\delta E^{(2)}$ behaves for $q^{+} \rightarrow 0$ like

$$
\delta E_{d i v}^{(2)}=-\frac{v^{+} e^{2}}{2} \int_{-\infty}^{\infty} \frac{d q^{+}}{q^{+^{2}}} \int_{-\pi / a}^{\pi / a} d q_{\perp} j(q) j(-q)=-\frac{v^{+} e^{2} a}{2} \int_{-\infty}^{\infty} \frac{d q^{+}}{q^{+^{2}}} \sum_{n} j_{n}(0) j_{n}(0),
$$

which diverges unless the net charge $j_{n}(0)$ on each (!) site is zero. However, a similar divergence (with opposite sign) arises from the instantaneous interaction, as can be directly read off from Eq. (14). The sum of the two terms is IR finite as long as the total (i.e. sum of charge on all sites) charge is zero ${ }^{6}$

$$
\delta E \equiv v^{+} P_{j j}^{-}+\delta E^{(2)}=\frac{e^{2} a}{2} \int_{-\infty}^{\infty} \frac{d q^{+}}{v^{+}} \int_{-\pi / a}^{\pi / a} d q_{\perp} \frac{\left(\frac{a}{2}\right)^{2} j(q) j(-q)}{\left(\frac{a q^{+}}{2 v^{+}}\right)^{2}+\sin ^{2}\left(\frac{a q_{\perp}}{2}\right)} .
$$

In particular, for the case of two (oppositely charged) point charges one finds in the limit $a \rightarrow 0$ the logarithmic interaction energy, characteristic for an Abelian gauge theory in $2+1$ dimensions

$$
\delta E=\frac{e^{2}}{2 \pi} \log \sqrt{R_{\perp}^{2}+R_{L}^{2}}+\text { const. . }
$$

At first this result (i.e. perfect cancellation of the IR singularity in perturbation theory) seems to contradict the general discussion of the non-compact formulation

6) This is not surprising since $Q E D_{2+1}$ confines. 
of gauge theories above, where it is shown that transversely separated charges need to be connected by a gauge string in order to cancel the IR divergences.

However, this apparent contradiction is resolved by the simple observation the $\perp$ lattice Hamiltonian for non-compact $Q E D_{2+1}$ coupled to external sources is, technically speaking, just a bunch of coupled shifted harmonic oscillators (the Hamiltonian is Gaussian!). This has two important consequences: First of all, for a shifted harmonic oscillators, the exact ground state energy is obtained already in $2^{\text {nd }}$ order perturbation theory. Therefore, the calculated ground state energy (18) is the exact one. Secondly, in the language of Fock space operators, the eigenstate of a shifted harmonic oscillator are coherent states, i.e. exponentials of raising operators.

Keeping these facts in mind, everything fits together: The coherent states, which are the eigenstates of the the $\perp$ lattice in the presence of the external source, accomplish the same effect as the exponentials of link fields, namely the act as soliton like operators which are necessary to cancel the small $q^{+}$divergence of the instantaneous self-interaction. Furthermore, the fact that we are dealing only with shifted harmonic oscillators in $Q E D_{2+1}$ also guarantees that we observed this cancellation already in perturbation theory - even though we did not construct these coherent states explicitly.

However, the bad news is that it is difficult to construct a Fock space basis containing exponentials of the gauge fields. ${ }^{7}$ Furthermore, when one cannot solve the problem exactly, it is very difficult to achieve this cancellation of IR singularities - unless one works with a basis of gauge invariant states, which is very hard in the non-compact formulation. As usual, in QCD, the situation is worse because the gauge fields themselves carry color-charge. Furthermore, if one tries to maintain gauge invariance then the ansatz $U_{\perp}=\exp \left(i g A_{\perp}\right)$ leads to $\frac{1}{g^{2}} \partial_{\mu} \exp \left(i g A_{\perp}\right) \partial^{\mu} \exp \left(-i g A_{\perp}\right)=\partial_{\mu} A_{\perp} \partial^{\mu} A_{\perp}+$ "higher orders" and these higher order terms make it again very difficult to quantize the theory. For these reasons, the non-compact formulation of the $\perp$ lattice has been abandoned.

\section{Color-Dielectric Formulation of the $\perp$ Lattice}

Naively, one might think that one possibility to introduce $U_{\perp} \in S U(N)$ fields would be to work with linearized general complex matrix fields and to add a potential $V_{\text {eff }}\left(U_{\perp}\right)$ that has a minimum for $U_{\perp} \in S U(N)$ (e.g. "Mexican hat" type potential). However, in the Fock expansion (an essential ingredient in the LF Hamiltonian formulation), the fields are usually expanded around the origin (or some other fixed value). Expanding around the origin makes no sense for a Mexican hat shaped potential since the origin corresponds to the false vacuum. On the other hand, expanding around any point along the minimum breaks the manifest global gauge symmetry. Therefore, this idea of adding a (Lagrange multiplier) effective self interaction to enforce the constraint must be abandoned as well.

7) Even in $Q E D_{2+1}$, the exact solution contains exponentials of $a^{\dagger}$ and thus leads to nonnormalizable states. 
A solution out of this dilemma is to work with blocked (smeared, averaged) degrees of freedom $\mathcal{M}$, which are obtained from the original $U_{\perp} \in S U(N)$ by averaging $U_{\perp} \mathrm{s}$ or strings of $U_{\perp} \mathrm{s}$ over some finite volume, e.g. by defining $\mathcal{M}=$ $\sum_{a v} U[5]$. The advantage of this procedure is that the smeared $\mathcal{M}$ s are no longer subject to the strict $S U(N)$ constraint.

The blocked theory is still equivalent to the original theory, provided the action for the $\mathcal{M}$ s contains an effective interaction defined by integrating out the $U_{\perp} \mathrm{s}$

$$
\exp \left[-V_{\text {eff }}(\mathcal{M})\right]=\int \mathcal{D} U_{\perp} \delta\left(\mathcal{M}-\sum_{a v} U\right) \exp \left[-S_{\text {canonical }}\left(U_{\perp}\right)\right] .
$$

The catch in the whole procedure is that $V_{\text {eff }}(\mathcal{M})$ as defined through Eq. (20) can be infinitely complicated and for its exact determination one would have to perform a path integral. However, for approximate calculations, one can always make an ansatz for $V_{\text {eff }}(\mathcal{M})$ and there exist various options to determine the parameters appearing in this ansatz. Note that a direct use of Eq. (20) to calculate $V_{\text {eff }}(\mathcal{M})$ within the LF framework does not seem to be possible: in order to evaluate the r.h.s. of Eq. (20) one needs to work with link-fields $U_{\perp} \in S U(N)$ and the difficulties in doing this were the main motivation to introduce the color-dielectric formulation in the first place. In the Euclidean, calculating $V_{\text {eff }}$ seems to be more straightforward, but for using it on the $\perp$ lattice, the issue arises of translating $V_{\text {eff }}$ from the Euclidean to the LF. ${ }^{8}$ An alternative procedure, based on covariance requirements, appears to be very promising. Due to lack of space, the reader is referred to Refs. $[7,5]$, where the procedure has been discussed in detail.

Acknowledgements: I would like to thank the organizers for the invitation and DFG for financial assistance. It is a pleasure to thank P. Griffin, B. vande Sande and S. Dalley for many enlightening discussions.

\section{REFERENCES}

1. Burkardt, M. Advances Nucl. Phys. 23, 1 (1995); Brodsky, S.J., Pauli, H.C., and Pinsky, S.S., Phys. Rept.301, 299 (1998).

2. Bardeen, W.A., and Pearson, R.B., Phys. Rev. D14, 547 (1976); Bardeen, W.A., Pearson, R.B., and Rabinovici, E., Phys. Rev. D21, 1037 (1980).

3. Griffin, P.A., in Theory of Hadrons and Light Front QCD, ed. S.D. Glazek, World Scientific, 1995, p. 240, hep-th/9410243.

4. Burkardt, M., in Theory of Hadrons and Light Front QCD, ed. S.D. Glazek, World Scientific, 1995, p. 233, hep-ph/9410219; Blunden, P.G. et al., hep-ph/9908067.

5. Dalley, S., and vande Sande, B., Phys. Rev. D59, 065008 (1999); Phys. Rev. Lett.82, 1088 (1999). Klindworth, B., and Burkardt, M., talk@ConfinementIII, hepph/9809283; Burkardt, M., and Klindworth, B., Phys. Rev. D55, 1001 (1997).

6. Burkardt, M., Phys. Rev. D47, 4628 (1993).

7. Dalley, S., these proceedings.

8) See Ref. [6] for a discussion of this issue in the context of scalar field theories. 